\title{
The Regular Projective Solution Space of the Figure-Eight Knot Complement
}

\author{
Saburo Matsumoto and Richard Rannard
}

\section{CONTENTS}

1. Introduction

2. Normal Surface Theory for the Figure-8 Knot Complement

3. The Regular Projective Solution Space

4. Dual Normal Surface Theory

5. Determining the Regular Projective Solution Space $\mathcal{R}$ for the Figure-8 Knot Complement

6. Homogeneous Surfaces and Vertices of $\mathcal{R}$

7. Symmetric Classes in $\mathcal{P}$

References
Matsumoto was partially supported by the Australian Research Council.

AMS Subject Classification: 57N35, 57M50

Keywords: projective solution space, normal surfaces, immersions
We define the regular immersed projective solution space for a triangulated 3-manifold, and completely determine it for the figure-8 knot complement. We show that there is a close relationship between vertices of this polytope and normal surfaces of a particularly simple form immersed in the manifold.

\section{INTRODUCTION}

Immersed normal surface theory has been the subject of considerable interest recently, as a means of extending the techniques and results of "classical" (embedded) normal surface theory to nonHaken manifolds. However, progress has been limited by a dearth of examples and results. This paper, which builds on the work in [Rannard 1999], determines the "large-scale" distribution of immersed normal surfaces in a particular example, the figure- 8 knot complement, using methods that apply to arbitrary manifolds. For another approach to immersed normal surface theory see [Letscher 1997].

\section{NORMAL SURFACE THEORY FOR THE FIGURE-8 KNOT COMPLEMENT}

We begin by describing normal surface theory as applied to the figure- 8 knot complement. We refer the reader to [Hemion 1992; Jaco and Tollefson 1995] for the theory in its full generality.

Let $M_{8}$ be the figure- 8 knot complement, and $T$ be the canonical ideal triangulation of $M_{8}$ given in [Thurston 1997]. We can form $T$ by taking two tetrahedra $\mathcal{T}_{1}$ and $\mathcal{T}_{2}$ and identifying the faces so as to match the edges as shown in Figure 1, and then deleting the single vertex:

We see that $T$ has two edges and four faces. We may view $M_{8}$ as a hyperbolic manifold with a single cusp. The link of the deleted vertex of $T$ is a torus. 

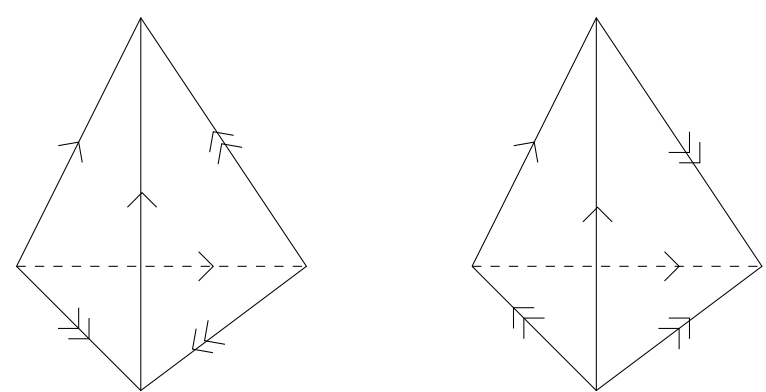

FIGURE 1. Ideal triangulation of the figure- 8 knot complement.

Any incompressible surface in a given triangulated manifold may be isotoped (or homotoped in case of an immersed incompressible surface) to meet the triangulation in a set of disks of a special type called normal disks. For more detailed discussions on the theory of normal surfaces, see [Hemion 1992].

Definition 2.1. A normal arc in a tetrahedron $\mathcal{T}$ is an arc properly embedded on a face of $\mathcal{T}$ whose two endpoints are on distinct edges of the face. A normal disk in $\mathcal{T}$ is a disk properly embedded in $\mathcal{T}$ that intersects each face of $\mathcal{T}$ in at most one normal arc.

It is easy to see that a normal disk in $\mathcal{T}$ is either

1. a triangle cutting off a vertex, or

2. a quadrilateral separating a pair of vertices

whose boundary edges are normal arcs. Normal disks of the first type are called T-disks, and those of the second are called $Q$-disks.

Definition 2.2. A normal surface in a triangulated 3manifold $M$ is an immersed surface that meets each tetrahedron in a set of normal disks. (A normal surface need not be connected or orientable.)
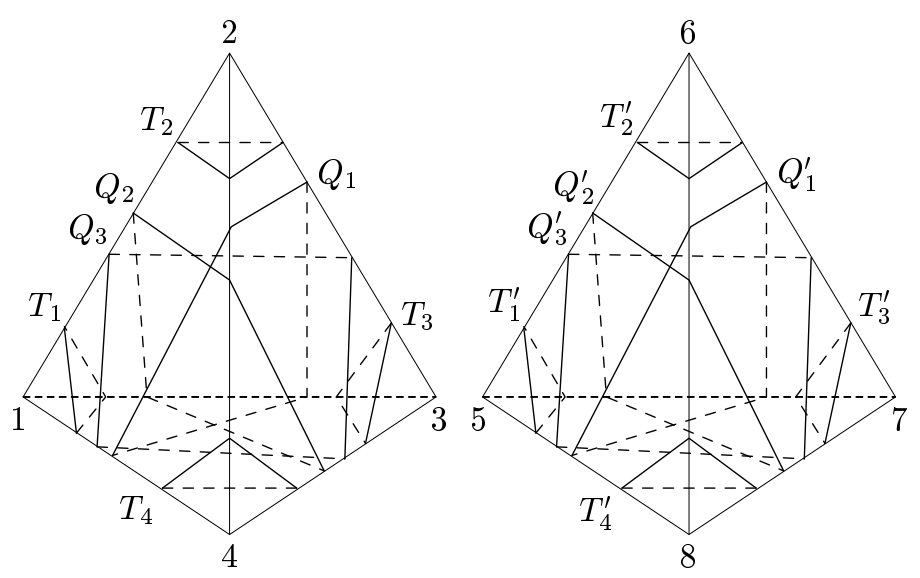

FIGURE 2. Normal disks in $M_{8}$.
Some edges of one tetrahedron $\mathcal{T}$ may actually be the same edge in $M$ after the faces of the tetrahedra are identified (e.g., in $M_{8}$, there are only two distinct edges). This may cause some normal arcs to begin and end on the same edge after the identification (as seen in $M_{8}$ ). Similarly, it is possible that a normal disk is not embedded but two of its edges are glued together. These phenomena do not affect our theory.

The normal disks for $M_{8}$ are shown in Figure 2. There are 7 such disks in each tetrahedron, making 14 in total. To each normal surface, we associate the vector

$$
\left(t_{1}, t_{2}, t_{3}, t_{4}, q_{1}, q_{2}, q_{3}, t_{1}^{\prime}, t_{2}^{\prime}, t_{3}^{\prime}, t_{4}^{\prime}, q_{1}^{\prime}, q_{2}^{\prime}, q_{3}^{\prime}\right) \in \mathbb{Z}^{14}
$$

where $t_{i}\left(q_{i}\right)$ is the number of normal disks of type $T_{i}\left(Q_{i}\right)$. The first seven belong to $\mathcal{T}_{1}$, and the last seven to $\mathcal{T}_{2}$. Each 14-tuple with nonnegative integer entries is called a class.

Suppose we try to reverse this operation, and ask what classes can correspond to surfaces in $M_{8}$. The class must satisfy some equations, called the matching equations: for each normal arc on each face of $T$, the number of normal disks meeting the face in that arc must be equal on each side of the face. (This is true even if a face of a tetrahedron $\mathcal{T}$ is glued to another face of $\mathcal{T}$; the matching equations still give a necessary condition.) In $M_{8}$, there are 12 matching equations, defining a cone of $\mathbb{Z}^{14}$, which we call the solution space. For $M_{8}$, this is the intersection of the positive coordinate half-planes and the subspace spanned by the vectors

$$
\begin{aligned}
& A=(1,1,1,1,0,0,0,1,1,1,1,0,0,0), \\
& B=(0,0,0,0,1,1,1,0,0,0,0,1,1,1), \\
& C=(0,0,1,1,1,0,2,0,0,1,1,1,0,2), \\
& D=(1,1,1,1,0,0,0,0,0,0,0,1,1,1) .
\end{aligned}
$$

Given a vector $v=\left(v_{1}, \ldots, v_{14}\right)$ in the solution space, we define the projectivization of $v$ to be the vector

$$
[v]=\frac{1}{\sum_{i=1}^{14} v_{i}}\left(v_{1}, \ldots, v_{14}\right) .
$$

Geometrically, this corresponds to projecting the vector $v$ onto the codimension 1 hyperplane defined by the equation

$$
x_{1}+\cdots+x_{14}=1 .
$$


The set of all vectors that project to the same projectivized vector is called a projective class. The set of all projective classes is called the projective solution space and denoted $\mathcal{P}$. We can visualize $\mathcal{P}$ as the intersection of the normal solution space with the codimension 1 hyperplane $x_{1}+\cdots+x_{14}=1$.

This projection both simplifies the space we seek and eliminate redundancy; for example, doubling a surface (making a parallel copy of a given surface) does not change the projective class while the class itself gets doubled. Clearly the projective solution space has dimension one less than the normal solution space; in this case it has dimension 3. Jaco and Rubinstein [1987] showed in that, for immersed surfaces in $M_{8}, \mathcal{P}$ is the three-dimensional solid octahedron with vertices the classes $[A],[B],[C],\left[C^{\prime}\right]$, $[D],\left[D^{\prime}\right]$, where $C^{\prime}$ and $D^{\prime}$ are defined in the next lemma (shown in Figure 3 and described below). The idea is as follows: clearly, $[A]$ and $[B]$ are independent, so pick two distinct points in $\mathbb{R}^{3}$ arbitrarily. $[C]$ and $\left[C^{\prime}\right]$ must then be chosen such that they satisfy the identity $C+C^{\prime}=A+2 B$. This means that the midpoint between $[C]$ and $\left[C^{\prime}\right]$ must be the point on the segment from $[A]$ and $[B]$ that represents the class $A+2 B$ (which, by the way, is not two-thirds of the way from $A$ to $B$ because of the projectivization). In other words, $[A],[B],[C]$, and $\left[C^{\prime}\right]$ are coplanar and generates a symmetric quadrilateral. The points $[D]$ and $\left[D^{\prime}\right]$ are not on this plane but need to be chosen so that the equation $D+D^{\prime}=A+B$ holds. So the segment between $[D]$ and $\left[D^{\prime}\right]$ passes through a point close to the center of the quadrilateral created by $[A],[B],[C]$, and $\left[C^{\prime}\right]$. Now, the symmetries between $[C]$ and $\left[C^{\prime}\right]$ and between $[D]$ and $\left[D^{\prime}\right]$ must be reflected in this object $\mathcal{P}$, so the space is a symmetric octahedron in the 3-dimensional space, as claimed above. The symmetry group of $\mathcal{P}$, then, is isomorphic to $\mathbb{Z}_{2} \oplus \mathbb{Z}_{2}$, with one generator flipping $[C]$ and $\left[C^{\prime}\right]$, the other flipping $[D]$ and $\left[D^{\prime}\right]$.

A vertex solution is a vector $V$ in the solution space that projects to a vertex of $\mathcal{P}$. The following result from [Jaco and Tollefson 1995] gives an algebraic characterization of vertex solutions.

Lemma 2.3. If a vector $V$ is a vertex solution, then the only solutions $A, B$ to the equation $m V=A+B$ are such that $A$ and $B$ are themselves multiples of $V$.

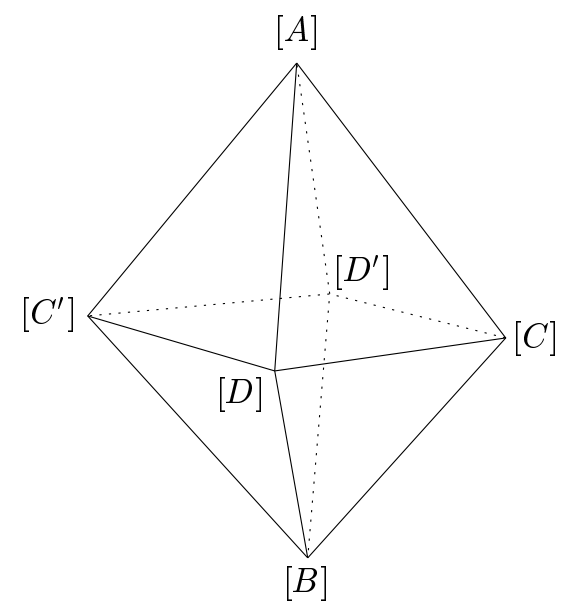

FIGURE 3. The projective solution space $\mathcal{P}$.

The vertices of $\mathcal{P}$ are the projectivizations of the classes

$$
\begin{aligned}
& A=(1,1,1,1,0,0,0,1,1,1,1,0,0,0), \\
& B=(0,0,0,0,1,1,1,0,0,0,0,1,1,1), \\
& C=(0,0,1,1,1,0,2, \quad 0,0,1,1,1,0,2), \\
& C^{\prime}=(1,1,0,0,1,2,0,1,1,0,0,1,2,0), \\
& D=(1,1,1,1,0,0,0,0,0,0,0,1,1,1), \\
& D^{\prime}=(0,0,0,0,1,1,1, \quad 1,1,1,1,0,0,0) .
\end{aligned}
$$

These may be interpreted geometrically as follows:

- $A$ consists of one each of all the T-disks. It is the link of the vertex, the torus neighborhood of the figure-8 knot.

- $B$ consists of one each of all the Q-disks.

- $C$ and $C^{\prime}$ are related by an involution $\sigma_{1}$ of $M_{8}$ that respects the triangulation.

- $D$ consists of one each of the T-disks in the first tetrahedron together with one each of the Q-disks in the second tetrahedron. $D$ and $D^{\prime}$ are related by a second involution $\sigma_{2}$ of $M_{8}$ that respects $T$.

These vectors are linearly dependent, with the identities mentioned in the description of the shape of $\mathcal{P}$; six vectors are required because no negative coordinates are allowed in these solution vectors.

We can picture $\mathcal{P}$ standing in $\mathbb{R}^{3}$ with $[A]$ as the top vertex, $[B]$ as the bottom vertex (and these two are on the $z$-axis), and $[C]$ and $\left[C^{\prime}\right]$ extending to the $y$-direction and $[D]$ and $\left[D^{\prime}\right]$ on the $x$-axis (see Figure 3 ). This space has natural symmetries (involutions) with respect to the $x, z$-plane and the $y, z$ plane. Note that we do not say that $[C]$ and $\left[C^{\prime}\right]$ are 
on the $y$-axis because $\mathcal{P}$ is not a regular octahedron and these two vertices are actually slightly "lower" than $[D]$ and $\left[D^{\prime}\right]$. We will make this concept more precise in Section 6.

The notion of projective solution spaces has been extensively studied; it proved to be a very powerful tool in [Jaco and Oertel 1984]; it is further discussed in [Tollefson and Wang 1996; Jaco and Tollefson 1995], and, more recently, [Tollefson 1998], in which the theory was developed using only the Q-disks. These papers suggest the importance of the vertices of $\mathcal{P}$ in normal surface theory.

Note that, while one can add two proper classes (say $V$ and $V^{\prime}$ ) simply as vectors, the sum of two projective classes $[V]$ and $\left[V^{\prime}\right]$ is not defined. Rather, all the points on $\mathcal{P}$ between $[V]$ and $\left[V^{\prime}\right]$ are described in the form $k[V]+(1-k)\left[V^{\prime}\right]$ for some $k \in$ $(0,1) \cap \mathbb{Q}$. The set of all such points on $\mathcal{P}$ will be referred to as the open segment between $[V]$ and $\left[V^{\prime}\right]$ on $\mathcal{P}$ and denoted $\overline{V V^{\prime}}$. One must also remember that, since these 6 "basis" vectors do not have the same number of disks, the projection is not "linear" in general, i.e.,

$$
\left[m V+n V^{\prime}\right] \neq \frac{m}{m+n}[V]+\frac{n}{m+n}\left[V^{\prime}\right]
$$

As real vectors, $[A]=\frac{1}{8} A,[B]=\frac{1}{6} B,[C]=\frac{1}{10} C$, and so forth. Hence, for instance, $[A+B]$ is not the midpoint of $[A]$ and $[B]$, but $[A+B]=\frac{4}{7}[A]+\frac{3}{7}[B]$. Similarly, it is easy to verify that

$$
\begin{aligned}
{\left[5 D+D^{\prime}\right] } & =\frac{11}{21}[A+2 D]+\frac{10}{21}[B+2 D], \\
\frac{1}{2}\left([C+3 D]+\left[C^{\prime}+3 D\right]\right) & =\frac{11}{31}[A+2 D]+\frac{20}{31}[B+2 D] .
\end{aligned}
$$

In particular, these equations show the following, which will be used later (see Figure 4).

Lemma 2.4. On the projective solution space $\mathcal{P}$, the points $[A+2 D],[B+2 D]$, and $\left[5 D+D^{\prime}\right]$ are collinear, and these points are coplanar with $[C+3 D]$ and $\left[C^{\prime}+3 D\right]$.

\section{THE REGULAR PROJECTIVE SOLUTION SPACE}

Every vector in the solution space may be represented as a collection of normal disks glued together, but this 2-dimensional structure is not necessarily an immersed surface. If a vector represents the set of normal disks produced when an immersed surface meets the tetrahedra of $M_{8}$, then it is called regular.

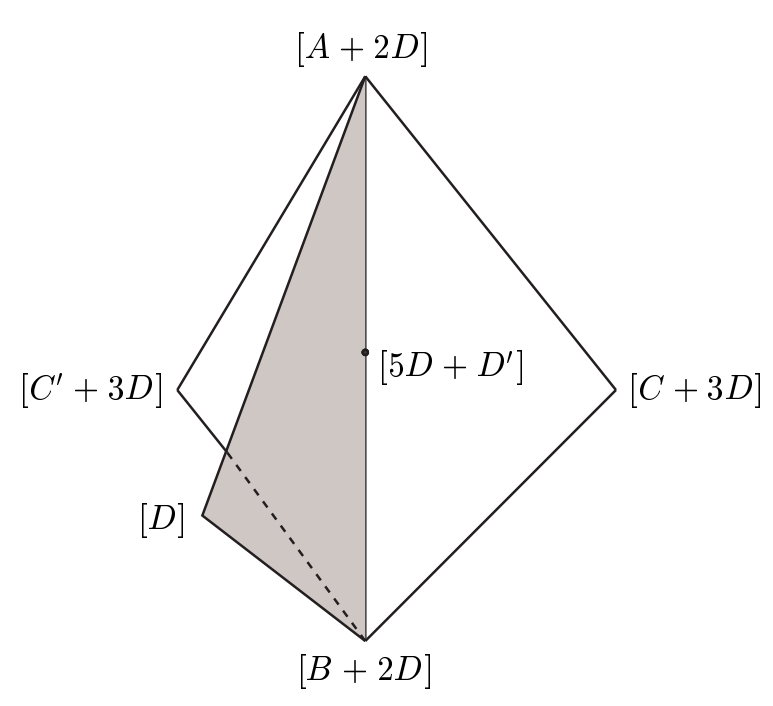

FIGURE 4. Around vertex $[D]$ of $\mathcal{P}$.

Irregular vectors correspond to collections of normal disks that cannot be glued together to give a surface. Irregular vectors exist in the solution space of $M_{8}$, and the central focus of this paper is to determine exactly which points of $\mathcal{P}$ represent regular classes.

At this point, it is helpful to introduce the dual structure of a 2-complex $(X, \mathcal{G})$ determined by a class $X$ of normal disks (in the solution space) and a given gluing $\mathcal{G}$. Pick the center point of each normal disk of $X$, and join two such points via a line segment (called a dual edge) if and only if the two normal disks share a common edge (i.e., glued together by $\mathcal{G}$ ). See Figure 5, which shows this structure for $M_{8}$. If one begins at the center point $p$ of some normal disk $D$ ( $Q_{a}$ in the figure) of $X$, proceeds to one

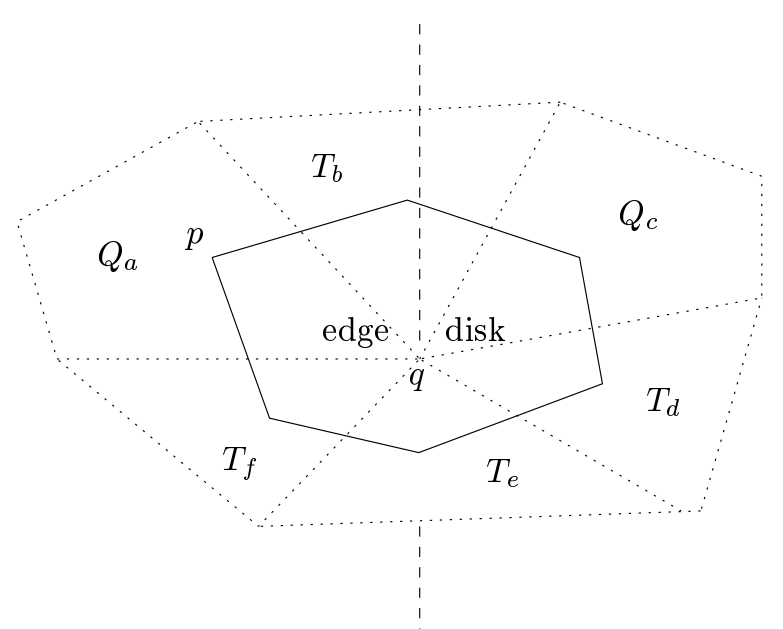

FIGURE 5. An edge disk. A dual loop (the thick solid line) is made up of 6 dual edges. The vertical dashed line is an edge of the triangulation. 


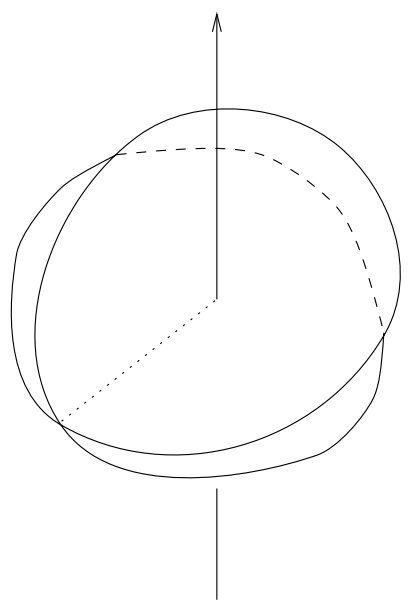

FIGURE 6. A typical branch point of order 2.

of the two adjacent normal disks sharing a vertex $q$ of $D$ (i.e., $T_{b}$ or $T_{f}$ ) and continues to move along the dual edge surrounding $q$, eventually one returns to $p$, but perhaps after going around $q$ more than once. The resulting path, a loop about $q$, is called a dual loop, and the compact polygon bounded by it is called a dual disk. (Hence, $(X, \mathcal{G})$ can be thought of as a union of these dual disks glued together along the dual edges.) This leads to the following definition.

Definition 3.1. If a dual loop goes around a vertex $q$ of $(X, \mathcal{G})$ exactly once, then the corresponding dual disk is called an edge disk; otherwise, $q$ is called a branch point of $(X, \mathcal{G})$.

Figure 6 shows a typical branch point of order 2 . Clearly, the 2-complex $(X, \mathcal{G})$ is an immersed normal surface if and only if every dual disk is an edge disk (see Figure 5). Note that an edge disk need not be embedded in the manifold, as before. The number of sides of this polygon obviously coincides with the degree of the edge, so it is possible to have a 1-gon or a 2-gon as an edge disk (although this does not happen in $M_{8}$ since all edges have degree 6). Also observe that each normal disk of $X$ is now subdivided into 3 or 4 quadrilaterals ( 3 for a T-disk, 4 for a Q-disk), each corresponding to a "corner" of the original T- or Q-disk. It is convenient to refer to these corners as T-corners and Q-corners, respectively, as we will see in the lemma below.

An example of an irregular vector is $D$, consisting of four T-disks of different types in one tetrahedron and three Q-disks of different types in the other.
One can easily verify (see the remark after the following lemma) that there is only one way to glue the 7 normal disks of $D$ together, which forces the disks to meet each edge of $M_{8}$ at a single point. In fact, it is evident that both of these points are branch points of order 2 .

More generally:

Lemma 3.2 [Aitchison et al. 1998]. If $S$ is a normal surface immersed in a triangulated manifold, viewed as the pre-image of the immersion (thus decomposed into T-disks and Q-disks), then each vertex of $S$ has an even number of Q-corners around it.

Note that we cannot say an "even number of Qdisks" since they may be different corners of one Q-disk.

For the class $D$ (and all its multiples), since the first tetrahedron can supply only T-disks and the second only Q-disks, as one goes around an edge (of the triangulation) to construct a dual disk, the T- and the Q-disks must alternate. But since the degree is 6 , one gets exactly 3 disks of each type, which shows why $D$ is irregular (as well as why every vertex of this 2-complex is a branch point as asserted above).

Definition 3.3. A projective class $[V]$ is regular if there is some regular class that projects to $[V]$. Otherwise, it is said to be irregular. The regular projective solution space $\mathcal{R}$ is the set of all regular projective classes in $\mathcal{P}$.

The regular projective solution space $\mathcal{R}$ is a convex sub-polytope of the projective solution space $\mathcal{P}$, since any linear combination of two regular classes represents a set of disconnected immersed surfaces. Note that points on the boundary of $\mathcal{R}$ need not a priori be in $\mathcal{R}$ itself. However, we will show that, at least for $M_{8}, \mathcal{R}$ is a compact space.

Section 5 deals with finding the shape of $\mathcal{R}$. Because of the two symmetries mentioned in Section 2, we need to consider only the subpolytope enclosed in the tetrahedron with vertices $A, B, C$, and $D$. The vertices $A, B$, and $C$ are all regular [Rannard 1999], but as noted above, $D$ projects to an irregular point. Since $\mathcal{R}$ is convex, there will be "critical points" on the edges connecting $[D]$ with the other three vertices of $\mathcal{P}$ marking the closest point a vector can be to $[D]$ without losing regularity. It is this ques- 
tion of "truncation" around $[D]$ (and $\left[D^{\prime}\right]$ ) that is central to determining the shape of $\mathcal{R}$ for immersed surfaces in $M_{8}$. In the following sections, we will introduce various techniques in search of the solution to this problem. First, in Section 4, we introduce an alternative normal surface theory dual to the standard theory described in Section 2. In Section 5 we give our main result - the complete description of $\mathcal{R}$ for the figure- 8 knot complement - which we obtained by applying the theories of Sections 2 and 4 using a computer. The last two sections of this paper present some geometric interpretations of the positions of the vertices.

\section{DUAL NORMAL SURFACE THEORY}

Since a regular surface meets the 1-skeleton of $M_{8}$ only in edge disks, it is natural to reverse the process and try to construct normal surfaces out of edge disks, instead of normal disks. We call this approach dual normal surface theory and develop it analogously to the standard normal surface theory.

First, we construct all the possible edge disks. The following lemma suggests the large number of possibilities we must consider.

Lemma 4.1. There are 128 possible edge disks for $M_{8}$, 64 about each edge.

Proof. Pick an edge $e$ of $M_{8}$ and picture 6 tetrahedra around $e$, as in the universal cover of $M_{8}$. Fix one of the tetrahedra as $\tau_{1}$ and label the others as $\tau_{2}, \tau_{3}, \ldots, \tau_{6}$, in order (say counterclockwise). Now, in $\tau_{1}$, there are two T-disks and two Q-disks that intersect $e$. This yields 4 possibilities in $\tau_{1}$ for an edge disk around $e$. Now, any one of these 4 disks gives rise to a normal arc in the face of $\tau_{2}$ glued to $\tau_{1}$, along which either a unique Q-disk or a unique $\mathrm{T}$-disk in $\tau_{2}$ can be glued. Once this is fixed, the same is true for $\tau_{3}, \tau_{4}$, and $\tau_{5}$. So we have counted $4 \times 2^{4}=64$ possible edge disks around $e$. Since every edge disk must have an even number of Q-corners, these 5 disks (or more precisely, the 5 corners) determine whether the corner in $\tau_{6}$ must be a Q-corner or a T-corner. Hence, there is only one choice for $\tau_{6}$, yielding 64 possible edge disks around $e$. Do the same with the other edge of $M_{8}$. Hence, there are 128 edge disks in total.

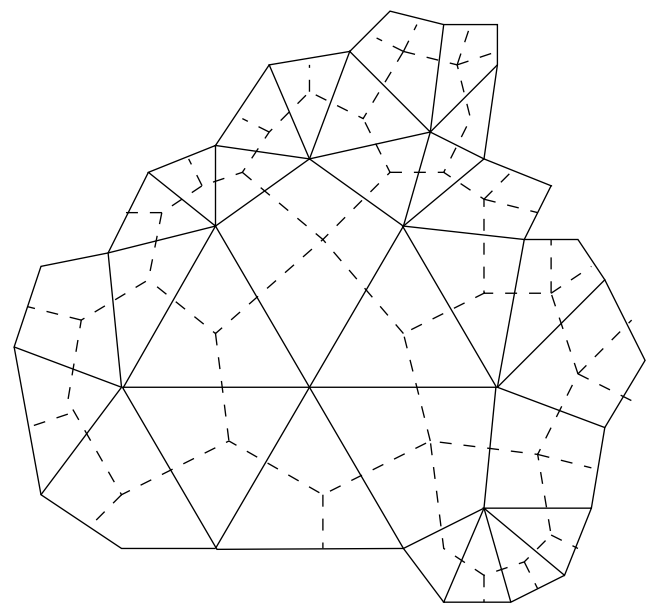

FIGURE 7. A normal surface viewed simultaneously as a set of normal disks glued together (solid lines) and as a set of edge disks glued together (dashed lines).

This list of 128 edge disks was first created by hand by the first author and later verified by a program written by the second author. Once the complete set of edge disks was generated, each edge disk was assigned a number.

When a regular surface is split up into a collection of edge disks, we see that two adjacent edge disks meet in a pair of normal disks (if a face of a tetrahedron is glued to another face of the same tetrahedron, an edge disk may join to itself-but this does not happen in $M_{8}$ ) sharing a common edge. See Figures 7 and 8.

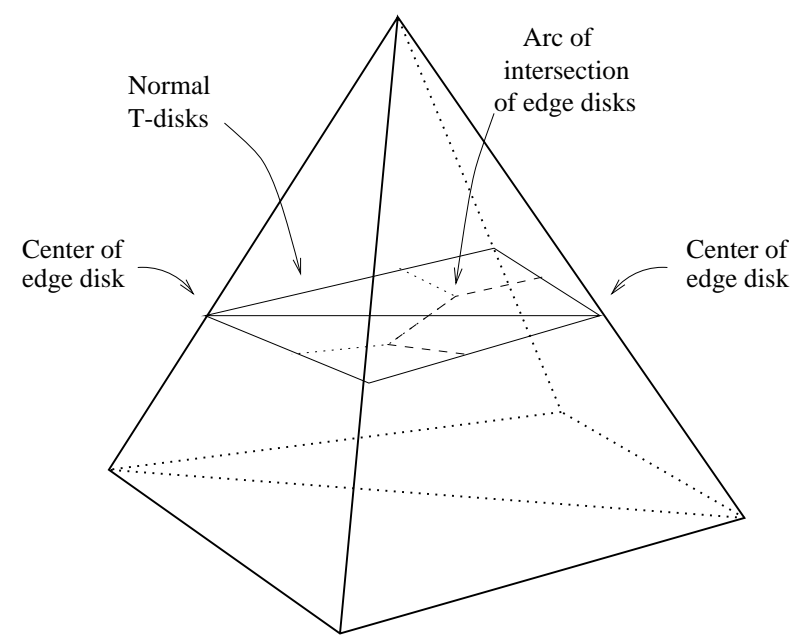

FIGURE 8. Two adjacent edge disks meeting in an arc. The boundary of the edge disks are drawn with dashed lines. 
Moreover, for each such pair of normal disks, the number of edge disks coming from one side must equal the number coming from the other. We obtain a set of equations, the dual matching equations, as a necessary condition for the surface to exist. Each pair of adjacent normal disks (normal disks which share a common edge) produces a dual matching equation, analogously to the way each normal arc gives rise to a matching equation in standard normal surface theory.

As in the standard normal surface theory, we identify each type of edge disk with a coordinate of a vector space over $\mathbb{R}$, so the dual matching equations define a subspace. The set of vectors in this subspace with all coordinates nonnegative is the dual solution space. Unfortunately, not every vector in the dual solution space need correspond to a regular surface, as shown below.

Since every regular surface is represented by a vector in both the standard normal solution space and the dual normal solution space, the question arises as to the relationship between these two vectors as well the two spaces. There is a natural linear map $U$ from the dual solution space to the standard solution space that answers part of this question, given as follows. Each edge disk $e_{i}$ is composed of corners of a fixed set of normal disks, say $w_{i_{1}}, \ldots, w_{i_{q}}$. The image of $e_{i}$ under the linear map is the vector representing

$$
\frac{1}{d_{1}} w_{1}+\cdots+\frac{1}{d_{q}} w_{q}
$$

where $d_{i}=3$ if $w_{i}$ is a T-disk and $d_{i}=4$ if $w_{i}$ is a Q-disk, as shown in Figure 9.

We extend this to a map $U$ on the whole dual solution space by linearity, which we call the dualto-standard map.
The image of the dual solution space under the dual-to-standard map is a cone, which we may convert into a polytope by intersecting the image of the dual solution space with the same hyperplane as in standard normal surface theory. We shall examine methods of computing this polytope in a subsequent section.

Another natural linear map derives from the following. Suppose an edge disk is described as

$$
\left[L_{1}, L_{2}, L_{3}, L_{4}, L_{5}, L_{6}\right]
$$

where the $L_{i}$ represent particular normal disk types (whose corners make up the edge disks). We can replace each $L_{i}$ by either a "Q" or "T" depending on whether it represents a Q-disk or a T-disk. In other words, one goes around the edge disk and writes down a "Q" if one comes to a Q-corner and a " $T$ " if a T-corner. An example of such a notation is QQQTQT. Two edge disks are combinatorially equivalent if their symbols are the same up to rotation and reflection. For immersed surfaces in $M_{8}$, there are 8 equivalance classes; see [Aitchison et al. 1998]. These 8 possible combinatorial types for edge disks are referred to as vertex types in [Aitchison et al. 1998], but we shall call them edge disk types here. We say a normal surface $S$ is homogeneous if all its edge disks are of the same edge type (combinatorially equivalent), and a point in $\mathcal{R}$ (i.e., a projectivized normal surface) is a homogeneous class if a homogeneous surface projects to it. As before, we can associate each combinatorial equivalence class of edge disks with a coordinate of a (8-dimensional) vector space, and construct the canonical projection. The image of the dual solution space under this map is a cone, which we may convert once again into a polytope by intersecting
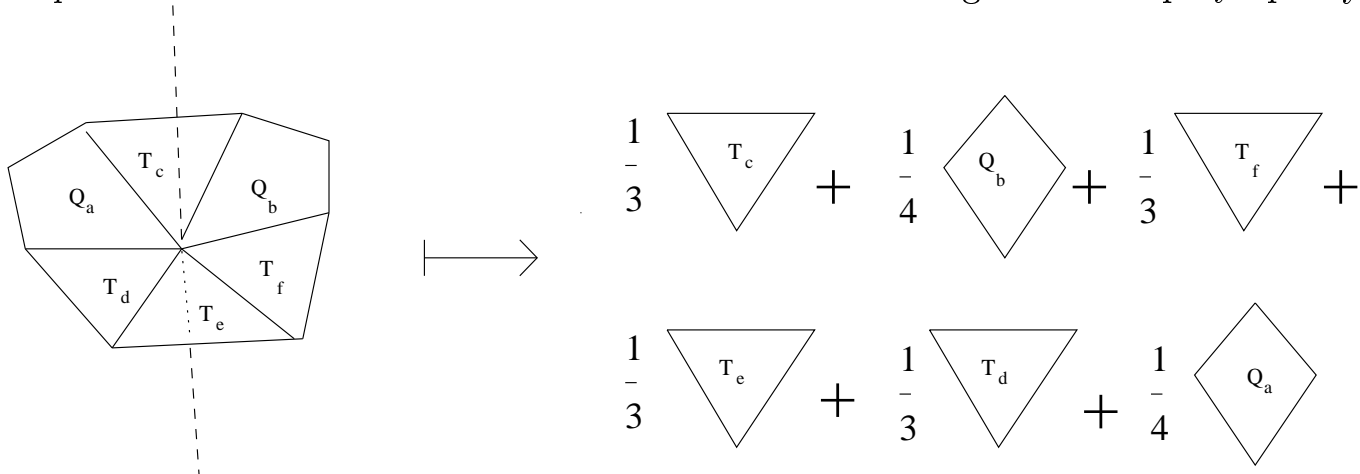

FIGURE 9. The action of the "dual-to-standard" map $U$ on an edge disk. 
this image with a hyperplane as in standard normal surface theory. Homogeneous surfaces clearly correspond to vertices of this polytope. For the figure- 8 knot complement, the vertices of this polytope have a strong connection with the vertices of the regular solution space, as outlined in Section 6. This polytope seems to be an interesting object, but we will not study it in detail in this paper; however, the following remarks seem appropriate to conclude this section.

We mentioned previously that not every vector in the dual solution space need correspond to a regular surface. It may happen that a set of edge disks match up along their boundaries, but the corners of the edge disks (corresponding to normal disks) don't match up to give a disk. This situation is analogous to the formation of branch points in the standard normal surface theory, so we call it a dual branch point. At a dual branch point a normal disk is replaced by some branched cyclic cover of the normal disk over its center.

An example of a case where a dual branch point must occur is the following. In [Aitchison et al. 1998] it is shown that the edge disk type QTTQTT cannot have homogeneous surfaces. However, there is a solution to the dual matching equations involving only edge disks of this type. As this solution cannot correspond to a normal surface, dual branch points will occur whenever the edge disks of the solution are glued together. The same is true for the edge disk type QQQQTT. Curiously, however, in each case the image of the dual solution under the map to the normal solution space is a regular class. This implies that the edge disks can be cut up along normal arcs and reglued to give a regular surface. In other words, although this map $U$ is very useful for practical computations (as we shall see in the following section), some nontrivial information gets lost by this map as well.

\section{DETERMINING THE REGULAR PROJECTIVE SOLUTION SPACE $\mathcal{R}$ FOR THE FIGURE-8 KNOT COMPLEMENT}

To determine $\mathcal{R}$, we first find a set of points known to be regular, and then show that all points outside the convex hull of this set are irregular. We make great use of the fact that $\mathcal{R}$ is convex.
The first step is accomplished by referring to the results in [Rannard 1999], the results of a computer search for regular surfaces using the Magma computational algebra system [Bosma et al. 1997]. From this we know that the classes $[A],[B],[C],[A+2 D]$, $[B+2 D]$ and $[C+2 D]$ are regular. The classes $\left[C^{\prime}\right],\left[A+2 D^{\prime}\right],\left[B+2 D^{\prime}\right],\left[C+2 D^{\prime}\right],\left[C^{\prime}+2 D\right]$, and $\left[C^{\prime}+2 D^{\prime}\right]$ are then regular by symmetry.

To show that points outside the convex hull are irregular, we use the fact that all regular vectors must lie inside the image of the dual solution space under $U$.

We now show how to compute this image. One potential method is to consider the standard normal solution space as a subspace of the dual solution space, and write the dual-to-standard map as the product of a bijective linear map with a projection onto this subspace. Then we could determine the image by using Fourier-Motzkin elimination on the dual matching equations and the inequalities $x_{i} \geq 0$, where the $x_{i}$ are the dual coordinates. However, for $\left(M_{8}, T\right)$ the difference in dimension between the normal solution space and the dual solution space was very large (4-dimensional versus 101-dimensional) and the number of intermediate vertices generated by Fourier-Motzkin elimination rises beyond the storage capacity of our computers, making this method infeasible. Instead we proceed by converting the question into a linear programming problem.

As noted in Section $3, \mathcal{R}$ is obtained from $\mathcal{P}$ by truncating the vertices $[D]$ and $\left[D^{\prime}\right]$. Knowing that $\mathcal{R}$ is convex, we can determine the truncation by finding the maximum value of the rational parameter $t$ such that $[V+t D]$ is regular, for various classes $[V]$. Our method is to view $t$ as the objective function in a linear programming problem.

The dual solution space and the dual-to-standard map are both linear. We can form the vector space

$\left(\begin{array}{c}\text { vector space } \\ \text { spanned by } \\ \text { edge disks }\end{array}\right) \times\left(\begin{array}{c}\text { vector space } \\ \text { spanned by } \\ \text { normal disks }\end{array}\right) \times\left(\begin{array}{c}\text { one-dim. } \\ \text { vector space } \\ \text { representing } t\end{array}\right)$.

The dual matching equations can be written as a set of linear equations relating the variables from the first factor, and the normal matching equations can be written as a set of linear equations among the 
variables of the second factor. The dual-to-standard map can be written as a set of linear equations relating the variables in the first and second factors. The equation $V(t)=[X+t D]$ can be written as a set of linear equations relating the second and third factors, with some constants. The whole set of linear equations can be gathered together and written

$$
M y=z, \quad y \geq 0
$$

where $y$ and $z$ are vectors, and $t$ occurs only as a linear factor. Our problem, as illustrated in Figure 10, can now be formulated as:

Maximize $t$ subject to the constraints $M y=z$, $y \geq 0$.

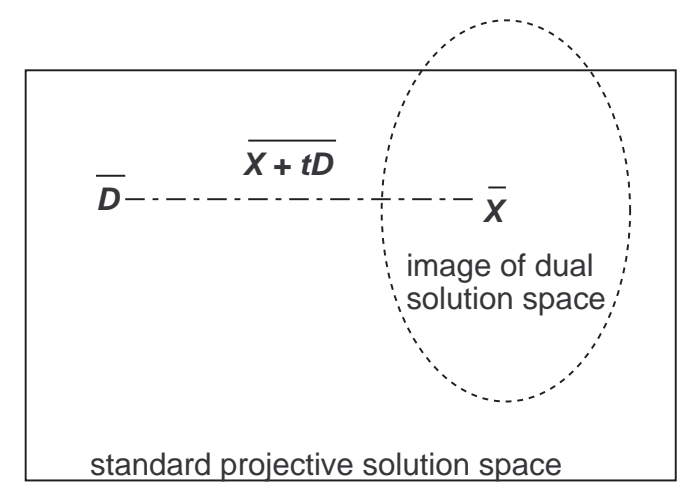

FIGURE 10. Finding the value of $t$ for which $[X+t D]$ moves out of the image of the dual solution space under the dual-to-standard map.

To implement this method, we have written a Mathematica file containing the following data:

- A list of 143 variables, labelled $x_{1}, \ldots, x_{143}$. The first 128 variables $x_{1}, \ldots, x_{128}$ correspond to the edge disks for $\left(M_{8}, T\right)$, the next 14 variables $x_{129}$, $\ldots, x_{142}$ correspond to the 14 normal disks, and the final variable $x_{143}$ represents the parameter $t$ to be maximized;

- The dual matching equations, expressed as a set of linear equations in $x_{1}, \ldots, x_{128}$;

- The normal matching equations, expressed as a set of linear equations in $x_{129}, \ldots, x_{142}$;

- The dual-to-standard map, expressed as a set of equations giving each of $x_{129}, \ldots, x_{142}$ as a linear combination of $x_{1}, \ldots, x_{128}$;

- A set of equations giving each of $x_{129}, \ldots, x_{142}$ in terms of $x_{143}$ and constants, representing the fact that we are looking for the vector $[X+t D]$.
To calculate the image of the dual solution space under the dual-to-standard map we use the LinearProgrammaing function in Mathematica, which solves the linear programming problem above. The objective function is $x_{143}$, which is maximized subject to the linear equations in the file. All the variables are kept nonnegative automatically. Coefficients are given as integers or fractions and all arithmetic is exact, avoiding roundoff errors or approximations. In general the resulting values for $x_{1}, \ldots, x_{143}$ will be nonintegral rational numbers, which become solution vectors after multiplying by a suitable large integer. This is not a problem since such vectors still project to points in the projective solution space.

The results are as follows:

Theorem 5.1. The regular projective solution space $\mathcal{R}$ is the convex hull of the points $[A],[B],[C],\left[C^{\prime}\right]$, $[A+2 D],[B+2 D],\left[A+2 D^{\prime}\right],\left[B+2 D^{\prime}\right],[C+2 D]$, $\left[C+2 D^{\prime}\right],\left[C^{\prime}+2 D\right]$, and $\left[C^{\prime}+2 D^{\prime}\right]$.

Proof. We use the method outlined above. Note first that of the six vertices of $\mathcal{P}$ only $[D]$ and $\left[D^{\prime}\right]$ are irregular. Using the process described above, we find that the classes $[A+t D],[B+t D]$, and $[C+t D]$ are irregular for $t>2$. The same process shows the class $\left[D^{\prime}+t D\right]$ is irregular for $t>5$, and hence by Lemma 2.4 the entire segment between $[A+2 D]$ and $[B+2 D]$ is in the boundary of $\mathcal{R}$. Finally, the class $[A+B+C+t D]$ is found to be irregular for $t>6$. We know $[A+B+C+6 D]$ is regular since $A+B+C+6 D=(A+2 D)+(B+2 D)+(C+2 D)$. The result then follows by the convexity of $\mathcal{R}$, the symmetries of $\mathcal{R}$ and the regularity of the vertices $[A],[B]$ and $[C]$.

The regular projective solution space $\mathcal{R}$ is shown in Figure 11.

A few questions naturally arise from this result. First, one can ask whether or not the regular projective solution space is strictly smaller than the image of the dual-to-standard map. In other words, is there a solution $V$ to the dual matching equations such that $U(V)$ does not satisfy the normal matching equations? As explained above, our method combines and solves the two sets of matching equations simultaneously and thus does not address this question in particular. We stated, at the end of Section 4 , that there are solutions to the dual equa- 


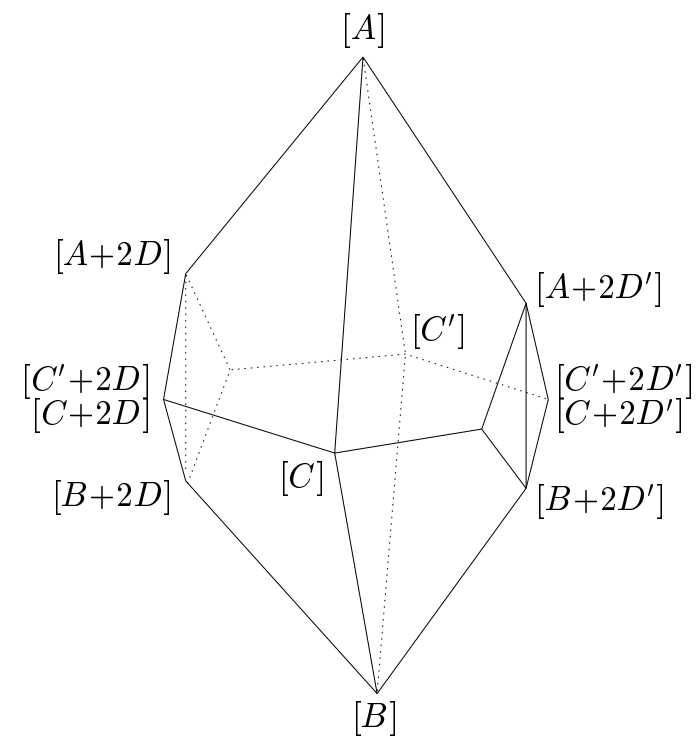

FIGURE 11. The regular projective solution space of the triangulated manifoled $\left(M_{8}, T\right)$.

tions that we know are irregular; however, these are mapped by $U$ to regular classes.

Another, perhaps more important, question is the following: is the regular projective solution space of a given triangulated manifold completely determined (as in the case of $M_{8}$ ) by the intersection of the image under the map $U$ of the dual solution space with the projective solution space? These two systems of equations give a lot of information, so certainly this is a possibility.

One by-product we obtain from the theorem is the following rather interesting result on what is referred to as virtually regular classes in [Rannard 1999].

Definition 5.2. A class is called virtually regular if some integer multiple of that class is regular.

Obviously the set of regular classes is a subset of the set of virtually regular classes.

Corollary 5.3. If $V$ is a virtually regular class in $M_{8}$, then its double $2 \mathrm{~V}$ is regular.

Proof. Suppose $V$ is a virtually regular vector. Using the identities $C+C^{\prime}=A+2 B$ and $D+D^{\prime}=A+B$, we can write $V$ in one of four forms: $V=v_{1} A+$ $v_{2} B+v_{3} C+v_{4} D, V=v_{1} A+v_{2} B+v_{3} C+v_{4} D^{\prime}$, $V=v_{1} A+v_{2} B+v_{3} C^{\prime}+v_{4} D$, or $V=v_{1} A+v_{2} B+$ $v_{3} C^{\prime}+v_{4} D^{\prime}$. By symmetry we need show the result only with one form; here we use the first.
Doubling both sides of the equation gives $2 \mathrm{~V}=$ $2 v_{1} A+2 v_{2} B+2 v_{3} C+2 v_{4} D$. We proceed to collect multiples of $A+2 D$. If $2 v_{1} \geq v_{4}$, the result is clearly the sum of two regular vectors, so we can glue the normal disks of $2 \mathrm{~V}$ together to give the (disconnected) sum of (at least) two regular surfaces. Otherwise, we have the equation $2 V=2 v_{2} B+2 v_{3} C+$ $2\left(v_{4}-2 v_{1}\right) D+2 v_{1}(A+2 D)$.

We now repeat the process for $2(B+2 D)$ and $2(C+2 D)$. If $v_{4}$ is even, we obtain

$2 V=2\left(v_{4}-2\left(v_{1}+v_{2}+v_{3}\right)\right) D+($ something regular $)$.

The restriction on the shape of the regular projective solution space $\mathcal{R}$ means $v_{4} \leq 2\left(v_{1}+v_{2}+v_{3}\right)$. If $v_{4}$ is odd, the result may contain the term $2 D$; however, if this occurs, the inequalities defining $\mathcal{R}$ imply there will always be a term $2 B$ or $2 C$ present. As we know $(2 B+2 D)$ and $(2 C+2 D)$ are both regular from [Rannard 1999], we conclude that $2 V$ is regular.

\section{HOMOGENEOUS SURFACES AND VERTICES OF $\mathcal{R}$}

There is a close relationship between the vertices of $\mathcal{R}$ and homogeneous surfaces in the standard solution space. In this section we examine this relationship more closely, and offer some alternative proofs of parts of Theorem 5.1 that illuminate the geometry of the situation and give more information on the surfaces in various classes.

Definition 6.1. Let $V$ be a regular class, and let $\left|Q_{V}\right|$ and $\left|T_{V}\right|$ be the numbers of Q-disks and T-disks in $V$, respectively. Define the vertex angle ratio of $V$ to be

$$
r_{V}=\frac{3\left|T_{V}\right|}{4\left|Q_{V}\right|},
$$

which is the ratio of the number of T-corners to the number of Q-corners. We set $r_{V}=\infty$ if $\left|Q_{V}\right|=0$.

Note that this ratio is well-defined on the projective class, so we may write $r_{V}$ to mean $r_{[V]}$. It is easy to observe that this ratio monotonically increases from 0 to $\infty$ as the class moves from $[B]$ up to $[A]$. In other words, we have defined a "height function" $r: \mathcal{P} \rightarrow \mathbb{R}^{+} \cup\{\infty\}$. The inverse image of some positive rational number under $r$ is a flat "horizontal" surface through $\mathcal{P}$. This map can be generalized to the solution space of any cell complex, as threequarters of the ratio of the total number of Q-disks to the total number of T-disks. 
Since each edge disk of an immersed normal surface must have an even number of Q-corners, i.e., 6, 4,2 , or 0 , it is easy to see the following:

Lemma 6.2. If $V$ is a homogeneous class, then $r_{V}$ must be $0, \frac{1}{2}, 2$, or $\infty$.

Hence, all homogeneous surfaces occur at the extreme points $[A],[B]$, and at only two levels (2 and $\left.\frac{1}{2}\right)$ of $\mathcal{P}$.

We will now use this result to find homogeneous classes. We begin with the vertices of $\mathcal{P}$. We already know $[D]$ and $\left[D^{\prime}\right]$ are not regular. On the other hand,

$$
r_{A}=\infty, \quad r_{B}=0, \quad r_{C}=r_{C^{\prime}}=\frac{1}{2},
$$

so $C$ and $C^{\prime}$ could be homogeneous. Indeed, it is shown in [Aitchison et al. 1998] that they are. The computer program of [Rannard 1999] has verified that the proper class $C$ has 13 surfaces altogether, and one of them ( $S_{7}$ of the type QQTQQT in [Aitchison et al. 1998]) is homogeneous.

Next, we look at the classes of the form $m\left[V_{1}\right]+$ $(1-m)\left[V_{2}\right], m \in(0,1) \cap \mathbb{Q}$, where the $V_{i}$ are the 6 vertices of $\mathcal{P}$ (the open segment $\overline{V_{1} V_{2}}$ ).

It is straightforward to check that on the open segment $\overline{A B}$, there are only two homogeneous classes. This follows from the fact that if $[V]=[m A+n B]$, where $m, n \geq 1$, then $r_{V}=\frac{1}{2}$ only when $[V]=$ $[A+2 B]$, and $r_{V}=2$ only when $[V]=[2 A+B]$. The fact that they are homogeneous is shown in [Aitchison et al. 1998]. Similarly, on $\overline{A C},[3 A+2 C]$ is the only possible homogeneous class (with $r_{V}=2$ ), but no surface has been explicitly found in this class.

Since $[A],[B],[C]$ and $\left[C^{\prime}\right]$ are known to be regular, the above mentioned classes do not contribute to finding new vertices of $\mathcal{R}$. To determine the truncation around $[D]$ and $\left[D^{\prime}\right]$, we now look at the edges involving $[D]$.

Theorem 6.3. The following is true concerning $\mathcal{P}$.

1. On the open segment $\overline{A D},[A+2 D]$ is the only homogeneous class.

2. On the open segment $\overline{B D}$, only $[B+2 D]$ is homogeneous.

Proof. For part 1, suppose $[V]=[m A+n D]$, where $m, n \geq 1$. Then,

$$
r_{V}=\frac{3 \cdot 4(2 m+n)}{4 \cdot 3 n}=\frac{2 m+n}{n}=1+\frac{2 m}{n} .
$$

Hence, $r_{V}$ cannot be $\frac{1}{2}$ but is 2 if and only if $m / n=$ $\frac{1}{2}$. Thus, $[V]=[A+2 D]$ is the only possible class, and this class contains Thurston's surface $S_{3}$ [Aitchison et al. 1998] of the type QTQTTT and thus is homogeneous.

The proof of part 2 is exactly the same. Figure 12 shows a regular surface (found by the second author's program) in the proper class $2(B+2 D)$, making $[B+2 D]$ a homogeneous class.

Here is an interpretation of the position of two of the vertices of $\mathcal{R}$.

Theorem 6.4. The point $[\alpha A+\delta D]$ is regular if and only if $\alpha / \delta \geq \frac{1}{2}$. The point $[\beta B+\delta D]$ is regular if and only if $\beta / \delta \geq \frac{1}{2}$.

Proof. Let $S$ be a regular surface that projects to the class $[\alpha A+\delta D]$. In each edge disk of $S$ there can be either 0 or 2 Q-corners, since all the Q-disks are in a single tetrahedron. Clearly the ratio $\alpha / \delta$ will be least when all the edge disks have exactly 2 Q-corners. Let $t$ denote the number of T-disks and $q$ the number of Q-disks. Then

$$
t=8 \alpha+4 \delta \text { and } q=3 \delta
$$

from the definitions of the classes $A$ and $D$. If there are $e$ edge disks, there are $4 e \mathrm{~T}$-corners and $2 e \mathrm{Q}-$ corners; since each T-disk has 3 corners and each Q-disk has 4, we find

$$
\frac{8 \alpha+4 \delta}{3 \delta}=\frac{t}{q} \geq \frac{4 e / 3}{2 e / 4}=\frac{8}{3},
$$

and hence

$$
\frac{\alpha}{\delta} \geq \frac{1}{2}
$$

The class $A+2 D$ corresponds to the case $\alpha / \delta=$ $\frac{1}{2}$, known to be regular from the program, and by taking sums of this class with $A$ we may construct a regular class corresponding to any rational value $\alpha / \delta \gtrless \frac{1}{2}$.

We use the same argument for classes $[\beta B+\delta D]$. If $S$ is a regular surface in such a class, we know each edge disk of $S$ can have either 4 or 6 Q-corners following the reasoning above, and the ratio $\frac{\beta}{\delta}$ is minimized if all edge disks have 4 Q-corners (i.e., of the type QQQTQT since all T-disks come from a single tetrahedron). Defining $t, q$ and $e$ as above, we have

$$
t=4 \delta, \quad q=6 \beta+3 \delta .
$$


There are $2 e \mathrm{~T}$-corners and $4 e \mathrm{Q}$-corners, so

$$
\frac{4 \delta}{6 \beta+3 \delta}=\frac{t}{q} \leq \frac{2 e / 3}{4 e / 4}=\frac{2}{3},
$$

by which we obtain

$$
\frac{\beta}{\delta} \geq \frac{1}{2}
$$

The equality holds for the class $2 B+4 D$. As we mentioned in Theorem 6.3, there is a (homogeneous) surface (Figure 12) in this class, so the result follows.

Remark 6.5. This argument actually shows more than the regularity: it implies that any surface in $[A+2 D]$ and in $[B+2 D]$ must be homogeneous, of the type QTQTTT and the type QQQTQT, respectively.

Another curious fact is that, while the proper class $2 B+4 D$ is regular, $B+2 D$ is known to be irregular; see [Rannard 1999]. It is interesting to investigate how and why the regularity varies among proper classes projecting to the same point in $\mathcal{P}$.

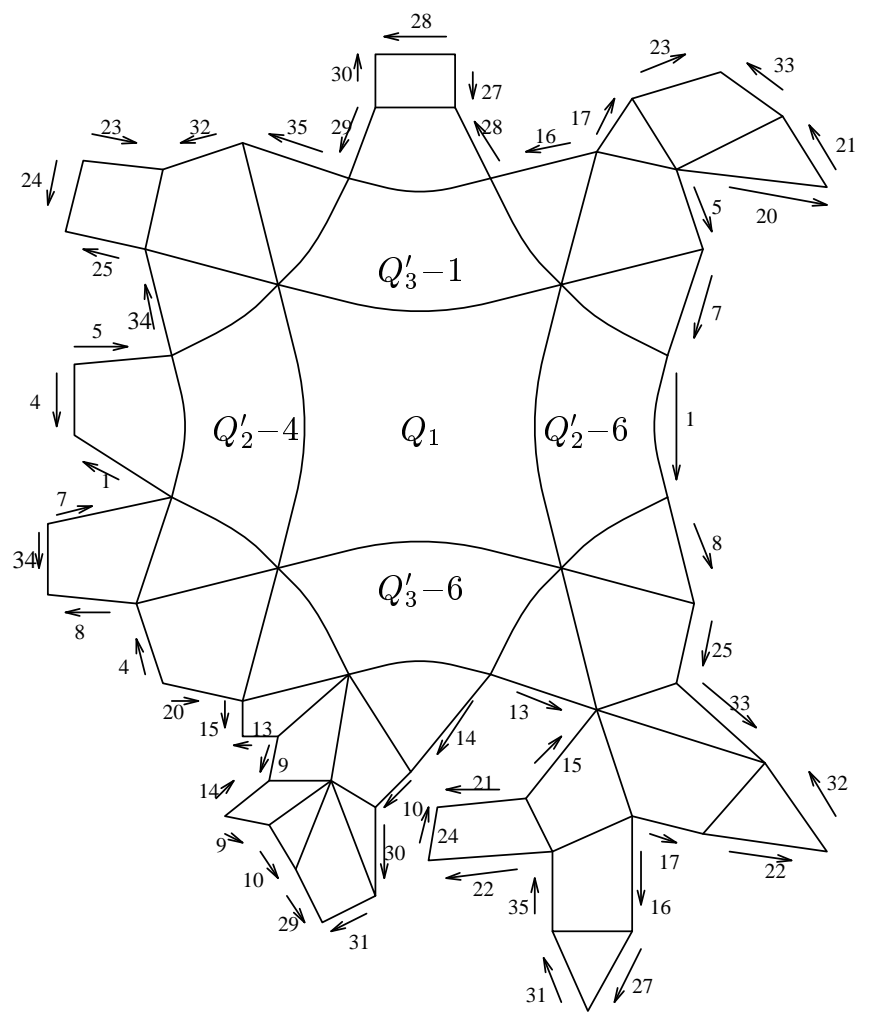

FIGURE 12. Homogeneous surface in $2(B+2 D)$.

There are no homogeneous classes in the open segment $\overline{C D}$ since $r_{V}$ is never 2 or $\frac{1}{2}$ in this segment.
In other words, while homogeneity gave a nice interpretation of the vertices of $\mathcal{R}$ on $\overline{A D}$ and $\overline{B D}$, it will not help us understand the vertex on $\overline{C D}$.

\section{SYMMETRIC CLASSES IN $\mathcal{P}$}

In order to study the regularity of classes near $[D]$ and $\left[D^{\prime}\right]$, we examine the 8 edge disk types a little more carefully (see Section 4). The following definition gives a way of understanding how the character of the edge disks of a surface close to $[D]$ differs from those of a surface in the plane containing $[A],[C]$, $[B]$ and $\left[C^{\prime}\right]$.

Definition 7.1. Let $v$ be an edge disk of a normal surface immersed in $M_{8}$. The edge disk type of $v$ is said to be symmetric if the number of Q-corners at $v$ coming from $\mathcal{T}_{1}$ (i.e., $Q_{1}, Q_{2}$, and $Q_{3}$ ) is equal to the number of $\mathrm{Q}$-corners coming from $\mathcal{T}_{2}$ (i.e., $Q_{1}^{\prime}, Q_{2}^{\prime}$, and $\left.Q_{3}^{\prime}\right)$. Otherwise, the edge disk type is nonsymmetric.

It is easy to see that we could use the number of T-corners in the definition instead. It is also trivial to verify that, of the 8 edge disk types possible, all but the types QQQTQT and QTQTTT are symmetric. Hence, if all edge disks of a surface $S$ are of the 6 symmetric types, the number of Q-disks from $\mathcal{T}_{1}$ is equal to the number of $\mathrm{Q}$-disks from $\mathcal{T}_{2}$, and the same holds for the number of T-disks. This observation gives us the following:

Lemma 7.2. Let $S$ be a normal surface immersed in $M_{8}$. If all the edge disks of $S$ are of symmetric types, then $S$ belongs to a class that is a linear combination of $A, B, C$, and $C^{\prime}$.

Proof. As noted above, such a surface $S$ has the same number of Q-disks (and T-disks) coming from each $\mathcal{T}_{i}$. If the class (say $V$ ) containing $S$ must have $D$ or $D^{\prime}$, these two numbers will not be equal since

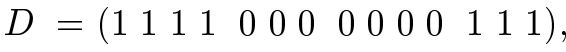

$$
\begin{aligned}
& D^{\prime}=\left(\begin{array}{lllllllllllll}
0 & 0 & 0 & 0 & 1 & 1 & 1 & 1 & 1 & 1 & 1 & 0 & 0
\end{array}\right) \text {. }
\end{aligned}
$$

This proves the lemma. Note that $V$ may have the same number of $D$ and $D^{\prime}$, but then the identity $D+$ $D^{\prime}=A+B$ allows $V$ to be written as a combination of $A, B, C$, and $C^{\prime}$.

For convenience, we introduce some notation. First, we count the number of nonsymmetric edge disks of 
a surface. Let $\alpha_{1}$ be the number of edge disks of the type $Q^{\prime} Q Q^{\prime} T Q^{\prime} T$, where the prime (') indicates that these Q-disks come from $\mathcal{T}_{2}$, and the others from $\mathcal{T}_{1}$. Let $\alpha_{2}$ be the number of edge disks of the type $Q Q^{\prime} Q T^{\prime} Q T^{\prime}$. Similarly, let $\beta_{1}$ be the number of edge disks of the type $Q^{\prime} T Q^{\prime} T T^{\prime} T$, and $\beta_{2}$ the number of edge disks of the type $Q T^{\prime} Q T^{\prime} T T^{\prime}$. For $i=1,2$, define $q_{i}$ to be the number of Q-corners from $\mathcal{T}_{i}$, and $t_{i}$ to be the number of T-corners from $\mathcal{T}_{i}$. Note the following facts.

Lemma 7.3. Let $|Q|$ (and $|T|)$ be the number of $Q$ disks (and T-disks) in a class $V$. Then $|Q|=\frac{1}{4}\left(q_{1}+\right.$ $\left.q_{2}\right)$ and $|T|=\frac{1}{3}\left(t_{1}+t_{2}\right)$. Moreover, $q_{2}-q_{1}=t_{1}-t_{2}=$ 0 if and only if $V$ is a linear combination of $A, B, C$, and $C^{\prime}$ only.

Proof. The values of $|Q|$ and $|T|$ are obvious. For the last statement, first suppose $V$ is a linear combination of $A, B, C$, and $C^{\prime}$. Each of these classes has the properties that $q_{1}=q_{2}$ and $t_{1}=t_{2}$, so the class $V$ also has these properties. Conversely, if $q_{1}=q_{2}$ and $t_{1}=t_{2}$ (actually one of these suffices), then clearly $V$ cannot have $D$ or $D^{\prime}$ (unless, as before, $V$ contains $D+D^{\prime}$, which can be written as $A+B$ instead).

We can now express the number of edge disks as

$$
|\mathcal{V}|=\frac{4|Q|+3|T|}{6}=\frac{q_{1}+q_{2}+t_{1}+t_{2}}{6}
$$

Lemma 7.4. The identity

$$
q_{2}-q_{1}=t_{1}-t_{2}=2\left(\left(\alpha_{1}-\alpha_{2}\right)+\left(\beta_{1}-\beta_{2}\right)\right)
$$

holds for any surface $S$ immersed in $M_{8}$.

Proof. As said before, the difference $q_{2}-q_{1}$ comes from nonsymmetric edge disks only. For each edge disk of the type $Q^{\prime} Q Q^{\prime} T Q^{\prime} T, q_{2}$ is increased by 3 while $q_{1}$ is increased by 1 . Similarly, each occurrence of $Q Q^{\prime} Q T^{\prime} Q T^{\prime}$ increases $q_{2}$ by 1 and $q_{1}$ by 3 . Finally, each occurrence of $Q^{\prime} T Q^{\prime} T T^{\prime} T$ increases $q_{2}$ by 2 while $Q T^{\prime} Q T^{\prime} T T^{\prime}$ increases $q_{1}$ by 2 . As other edge disks are irrelevant, we have

$$
\begin{aligned}
q_{2}-q_{1} & =\left(3 \alpha_{1}+\alpha_{2}+2 \beta_{1}\right)-\left(\alpha_{1}+3 \alpha_{2}+2 \beta_{2}\right) \\
& =2 \alpha_{1}-2 \alpha_{2}+2 \beta_{1}-2 \beta_{2} \\
& =2\left(\left(\alpha_{1}-\alpha_{2}\right)+\left(\beta_{1}-\beta_{2}\right)\right) .
\end{aligned}
$$

A similar calculation shows that

$$
t_{1}-t_{2}=2\left(\left(\alpha_{1}-\alpha_{2}\right)+\left(\beta_{1}-\beta_{2}\right)\right) .
$$

These lemmas give some insight into the shape of the regular projective solution space $\mathcal{R}$ for $M_{8}$.

Theorem 7.5. Let $V$ be the class expressed as $V=$ $m D+n D^{\prime}$, where $m, n$ are positive integers. If $V$ is regular, then $m / n \leq 5$.

Proof. Suppose $V$ is regular, and $S$ is a surface in $m D+n D^{\prime}$. By the definitions of $D$ and $D^{\prime}$, we get $q_{1}=12 n, q_{2}=12 m, t_{1}=12 m, t_{2}=12 n$, and $|\mathcal{V}|=4 m+4 n$. Therefore,

$$
q_{2}-q_{1}=12 m-12 n=2\left(\left(\alpha_{1}-\alpha_{2}\right)+\left(\beta_{1}-\beta_{2}\right)\right),
$$

from which we get

$$
6 m-6 n=\left(\alpha_{1}-\alpha_{2}\right)+\left(\beta_{1}-\beta_{2}\right) \leq 4 m+4 n .
$$

Hence,

$$
2 m \leq 10 n, \text { or } m / n \leq 5
$$

Remark 7.6. The equality holds when

$$
\begin{aligned}
V=5 D+D^{\prime} & =\left(D+D^{\prime}\right)+4 D=(A+B)+4 D \\
& =(A+2 D)+(B+2 D) .
\end{aligned}
$$

We know that $[A+2 D]$ and $[B+2 D]$ are regular, so the theorem shows that $\left[5 D+D^{\prime}\right]$ is the sharp bound on this segment between $[D]$ and $\left[D^{\prime}\right]$. In addition, any surface in $\left[5 D+D^{\prime}\right]$ has the property that every edge disk is of the type $Q^{\prime} Q Q^{\prime} T Q^{\prime} T$ or $Q^{\prime} T Q^{\prime} T T^{\prime} T$ and both types must occur.

The same type of argument shows the following; we omit the proof, which is very similar to the proof above.

Theorem 7.7. Let $V$ be the class expressed as $V=$ $m C+n D$, where $m, n$ are positive integers. If $V$ is regular, then $n / m \leq 3$.

Of course, we already know this by Theorem 5.1, which says that $V$ is regular if and only if $\frac{n}{m} \leq 2$. The reason we state it here is that this kind of argument gives many properties of prospective surfaces in these classes and often enables us to determine the regularity without solving a large system of equations by computer. (We eventually determined by hand that $[C+3 D]$ is irregular.) In fact, because of this last theorem and the fact that $[C+3 D]$ and $\left[C^{\prime}+3 D\right]$ are coplanar with the known vertices $[A+2 D],\left[D^{\prime}+5 D\right]$, and $[B+2 D]($ Lemma 2.4), we had once conjectured that these two points are the missing vertices of $\mathcal{R}$. In this context, it is very interesting and surprising that $[C+2 D]$, not $[C+3 D]$, 
is the vertex between $[C]$ and $[D]$. Perhaps this is due to the fact that $[C+2 D],\left[C+2 D^{\prime}\right],[A+2 D]$ and $[B+2 D]$ lie on an affine plane parallel to the homogeneous plane containing $[A],[C],[B]$ and $\left[C^{\prime}\right]$. Since the plane is affine, the convexity arguments used above fail.

\section{REFERENCES}

[Aitchison et al. 1998] I. R. Aitchison, S. Matsumoto, and J. H. Rubinstein, "Surfaces in the figure-8 knot complement", J. Knot Theory Ramifications 7:8 (1998), $1005-1025$.

[Bosma et al. 1997] W. Bosma, J. Cannon, and C. Playoust, "The Magma algebra system, I: The user language", J. Symbolic Comput. 24:3-4 (1997), 235265. See http://www.maths.usyd.edu.au:8000/comp/ magma/Overview.html.

[Hemion 1992] G. Hemion, The classification of knots and 3-dimensional spaces, Oxford University Press, New York, 1992.

[Jaco 1987] W. Jaco, 1987. unpublished personal notes.
[Jaco and Oertel 1984] W. Jaco and U. Oertel, "An algorithm to decide if a 3-manifold is a Haken manifold", Topology 23:2 (1984), 195-209.

[Jaco and Tollefson 1995] W. Jaco and J. L. Tollefson, "Algorithms for the complete decomposition of a closed 3-manifold", Illinois J. Math. 39:3 (1995), 358406.

[Letscher 1997] D. Letscher, Immersed normal surfaces and decision problems for 3-manifolds, Ph.D. thesis, University of Michigan, Ann Arbor, MI, 1997.

[Rannard 1999] R. Rannard, "Computing immersed normal surfaces in the figure-eight knot complement", Experiment. Math. 8:1 (1999), 73-84.

[Thurston 1997] W. P. Thurston, Three-dimensional geometry and topology, Vol. 1, Princeton University Press, Princeton, NJ, 1997.

[Tollefson 1998] J. L. Tollefson, "Normal surface $Q$ theory", Pacific J. Math. 183:2 (1998), 359-374.

[Tollefson and Wang 1996] J. L. Tollefson and N. Wang, "Taut normal surfaces", Topology 35:1 (1996), 55-75.

Saburo Matsumoto, Department of Mathematics, Belhaven College, Jackson, MS 39202, United States (smatsumoto@belhaven.edu)

Richard Rannard, School of Mathematics and Statistics, University of Sydney, N.S.W. 2006, Australia (richardr@maths.usyd.edu.au)

Received September 12, 1998; accepted in revised form June 9, 1999 